\section{Enge Verwandte: Spulwurm und Milbe}

\author{
Spulwurmbefall kann epidemiologischen Studien zufolge vor Asthma \\ und Atopie schützen oder Asthma und Atopie fördern. Diese \\ widersprüchlichen Ergebnisse beruhen möglicherweise auf Kreuz- \\ reaktivitäten zwischen Spulwurm- und Milbenallergenen.
}

E in international zusammengesetztes Forscherteam nahm in einer Experimentalstudie die IgE-Kreuzreaktivität von einem Extrakt des Spulwurms Ascaris lumbricoides und Extrakten der beiden Milbenarten Dermatophagoides pteronyssinus und Blomia tropicalis unter die Lupe. Dazu wurden die Seren von 24 Asthmapatienten der kolumbianischen Stadt Cartagena entsprechend ihrem Anti-IgETiter gegen $A$. lumbricoides, $D$. pteronyssinus sowie $B$. tropicalis und die rekombinanten Allergene rBlot 10, rBlo t 13 sowie rABA-1 in Gruppen gepoolt. Bei Blo t 10 handelt es sich um ein wichtiges Allergen von B. tropicalis, dessen Aminosäuresequenz zu 73\% mit der des Tropomyosins von Spulwürmern identisch ist, bei Blo t 13 um ein fettsäure- bindendes Protein. ABA-1 schließlich ist ein wichtiges Allergen von Spulwurmarten, das zur Familie der Polyproteinallergene gehört und über fettsäurebindende Eigenschaften verfügt. Im ELISA sowie im Immunoblot-IgE-Inhibitionstest wurden die Poolseren dann auf Kreuzreaktionen der darin enthaltenen IgE-Antikörper mit dem Spulwurmextrakt und den beiden Milbenextrakten getestet. Als Bindungskonkurrenten zu den in den Extrakten enthaltenen Allergenen wurden die rekombinanten Allergene eingesetzt.

Im Spulwurmextrakt wurden mindestens zwölf IgE-Antikörper-bindende Proteinbereiche nachgewiesen. Die Extrakte von $B$. tropicalis und D. pteronys sinus inhibierten zu 83,3\% bzw. 79\% die IgE-Bindung des Spulwurmextrakts, während dieser die IgE-Bindung von $B$. tropicalis und D. pteronyssinus zu 58,3\% bzw. 79,3\% inhibierte. Als kreuzreagierende Allergene wurden unter anderem Tropomyosin und Glutathion-S-Transferase identifiziert. Das rekombinante Spulwurmallergen rABA-1 zeigte keine Interaktion mit den gegen Milbenallergene gerichteten Antikörpern.

Fazit: Extrakte von Spulwurm und Milbe zeigen eine hohe Kreuzreaktivität. Damit könnte der Triggereffekt eines Spulwurmbefalls auf die Entwicklung von Asthma und Atopie erklärt werden. In serologischen Tests zum Nachweis einer Infektion mit Ascaris sp. sollte zum Ausschluss von unerwünschten Kreuzreaktionen das mit Anti-Milben-IgE nicht wechselwirkende Allergen ABA-1 als Reagens verwendet werden.

$b k$

Acevedo $\mathbf{N}$ et al. IgE cross-reactivity between Ascaris and domestic mite allergens: the role of tropomyosin and the nematode polyprotein ABA-1. Allergy 2009; 64: 1635-43

\title{
IL-4-Blockade kann Entzündung verstärken
}

\section{Die TH2-Interleukine IL-4 und IL-13 sind wichtige Mediatoren aller- gischer Entzündungsreaktionen. Doch eine therapeutische Blockade dieser Interleukine könnte offenbar eher schaden als nützen.}

A n einem Mausmodell sollte untersucht werden, wie sich ein Fehlen von IL-4 und IL-13 auf die TH17-Antwort auswirkt. TH17-Zellen bilden unter anderem die Interleukine IL-17A und IL-17F, die eine verstärkte Einwanderung von Neutrophilen ins Entzündungsgebiet bewirken. Patienten mit atopischem Ekzem oder Asthma weisen häufig erhöhte IL17-Spiegel auf.

Studien an KnockoutMäusen sollen helfen, die Rolle der Interleukine IL-4 und IL-13 als Allergiemediatoren zu verstehen.
Für den Tierversuch wurden Wildtyp-Mäuse, IL-4-Knockout(KO)-Mäuse, IL-13-KO-Mäuse und IL-4/IL-13-Doppel-KO-Mäuse mit Ovalbumin epikutan sensibilisiert und dann einer Atemwegsprovokation mit Ovalbumin ausgesetzt. Im Vergleich zu den Wildtyp-Mäusen zeigten die epikutan sensibilisierten IL4/IL-13-Doppel-KO-Mäuse eine verschlechterte Immunantwort bei nicht mehr nachweisbaren IgE-Spiegeln gegen Ovalbumin und einer herabgesetzten Eosinophilen-Infiltration der sensibilisierten Haut. Die $\mathrm{CD}^{+}$-Einwanderung in die Haut war nicht beeinträchtigt. Die Mäuse produzierten deutlich mehr IL17A. IFN- $\gamma$ und IL-5 wurden dagegen in normalen Konzentrationen freigesetzt. Die Atemwegsprovokation mit Ovalbumin verursachte bei den Doppel-KO-
Mäusen eine Aufregulation der IL-17 mRNA-Expression im Lungengewebe. Die bronchoalveoläre Lavage offenbarte einen Anstieg der Neutrophilen und eine entzündliche Reaktion mit einer Infiltration durch mononukläre Zellen bei nicht nachweisbaren Eosinophilen. Die bronchiale Hyperreagibilität konnte durch eine IL-17-Blockade aufgehoben werden. Eine Herabregulation der IL-17Produktion erfolgte durch IL-4, nicht dagegen durch IL-13.

Fazit: Im Mäuseversuch führt die epikutane Sensibilisierung mit Ovalbumin in Abwesenheit von IL-4 und IL-13 zu einer verstärkten systemischen TH17Antwort. Gleiches gilt für die Lunge nach Atemwegsprovokation mit Ovalbumin. Die IL-17-Produktion kann durch IL-4 herabreguliert werden. $\quad b k$

He $\mathbf{R}$ et al. Exaggerated IL-17 response to epicutaneous sensitization mediates airway inflammation in the absence of IL-4 and IL-13. J Allergy Clin Immunol 2009; 124: 761-70 